\title{
Cystic echinococcosis in slaughtered cattle in Sardinia: a retrospective epidemiological study and spatial analysis
}

\author{
Diego Brundu ${ }^{1}$, Daniela Aloi ${ }^{2}$, Sandro Rolesu², Toni Piseddu ${ }^{1}$, Giovanna Masala ${ }^{1}$ \\ ${ }^{1}$ Istituto Zooprofilattico Sperimentale della Sardegna, Centro di Referenza Nazionale per l'Echinococcosi \\ (CeNRE), Sassari, Italy; ${ }^{2}$ Istituto Zooprofilattico Sperimentale della Sardegna, Osservatorio Epidemiologico \\ Veterinario Regionale (OEVR), Cagliari, Italy
}

\begin{abstract}
Cystic echinococcosis (CE) in cattle was found in 246 out of all 377 municipalities in Sardinia, Italy. Out of 32,685 bovines slaughtered in Sardinia in 2009, 1,360 were found to be positive for CE with a registered average prevalence of $4.2 \%$. Of these animals, $896(66 \%)$ had lived on the same farm from birth to slaughter, thus linking the infection to the farm with certainty, while $413(30 \%)$ had lived on two different farms (one transfer) and 51 (4\%) on three (two transfers). As it was not possible to assess in which farm the animals acquired the infection, all farms having kept infected cattle were considered as suspected sources of CE infection. Based on this classification, 534 farms were listed as definitely infected with a further 495 suspected to also be infected. Scan statistics was used with the Bernoulli model to detect and evaluate clusters of infected farms and also clusters of "non-cases". For the spatial analysis, 1,029 farms $(534+495)$ were considered as positive with the number of non-infected farms from which negative results were available $(8,457)$ as controls. A most likely cluster was detected at latitude $39.47861 \mathrm{~N}$ and longitude $8.58216 \mathrm{E}$ in a centroid of $97.92 \mathrm{~km}$ radius and a secondary cluster was detected at latitude $40.58890 \mathrm{~N}$ and longitude $8.98400 \mathrm{E}$ in a centroid of $15.44 \mathrm{~km}$ radius. To address the issue of sensitivity and consistency of the results, we ran multiple scans with various max-sizes as this allowed us to achieve more valid, consistent results and to highlight the core clusters.
\end{abstract}

Keywords: cystic echinococcosis, slaughtered cattle, geographical information systems, spatial analysis, Italy.

\section{Introduction}

Cystic echinococcosis (CE), caused by Echinococcus granulosus, is an important zoonotic, parasitic infection causing morbidity and mortality in humans apart from significant economic losses in livestock (Budke et al., 2006). The parasite lifecycle includes dogs and other canids as definitive hosts, whilst sheep and numerous ungulates (cattle, goats, pigs, etc.) are intermediate hosts harbouring the hydatid cysts (Moro and Schantz, 2006; Craig et al., 2007). Dogs usually acquire the infection from hydatid-carrying livestock (especially sheep) as a result of being fed with infested offal (liver and lungs) by owners who practise homeslaughter (McManus et al., 2003).

$\mathrm{CE}$ is a considerable public health concern with a world-wide distribution. The endemic regions are in western and central Asia, People's Republic of China, South America, Australia, the northern and eastern

\footnotetext{
Corresponding author:

Giovanna Masala

Istituto Zooprofilattico Sperimentale della Sardegna

Centro di Referenza Nazionale per l'Echinococcosi (CeNRE)

Via Duca degli Abruzzi 8, 07100 Sassari, Italy

Tel. +39079 289 2325; Fax +39079 2892324

Email: giovanna.masala@izs-sardegna.it
}

parts of Africa as well as many countries of the Mediterranean basin (Seimenis, 2003; Jenkins et al., 2005; Romig et al., 2006). For E. granulosus, the highest prevalence rates among humans and animals occur where livestock production is extensive, e.g. large-scale sheep farming where many dogs are kept (e.g. for guarding the livestock) and where they have access to carcasses of dead livestock or offal after uncontrolled slaughter (Schantz et al., 1995). For these reasons the island of Sardinia, located in the western Mediterranean, is a suitable site for the study of this important zoonosis (Scala et al., 2006).

Recently, the prevalence of CE in Sardinia has been reported to be the following: sheep $75 \%$, goats $24 \%$, cattle $41.5 \%$, home-slaughtered pigs $11 \%$ and horses 4\% (Garippa, 2006). As epidemiological information is obtained from research results rather than proper surveys, the real epidemiological situation remains largely unknown. Indeed, the lack of official data and the poor level of reporting, both for animals and humans, do not allow a clear and detailed picture of the occurrence of CE in Italy (Garippa, 2006). The 2003/99/EC directive forms the basis for data on zoonoses collected throughout the European Union (EU) and each country reports to the EU Commission on an annual basis. CE is included in list A of Annex 
I, which determines the infections in EU that must be monitored on a mandatory basis. In December 2008, the Department of Hygiene, Health and Welfare of the Sardinian Region, with assistance of the Italian National Reference Centre for Echinococcosis (CeNRE), issued a legislative act about E. granulosus monitoring of all slaughtered cattle in order to compensate for the lack of official data from the abattoirs. This legislative act established a data flow from the local veterinary services to the CeNRE.

Our study was carried out to provide a detailed picture of the distribution of CE in Sardinian cattle farms based on geographic data extracted from national bovine identification database (BDN). We analysed available official data of all cattle slaughtered in Sardinian abattoirs with the objective to find the prevalence of hydatid infection in different categories of slaughtered cattle and the distribution of infected farms.

\section{Materials and methods}

\section{Study area}

The island of Sardinia has an area of $24,000 \mathrm{~km}^{2}$ and is divided into 377 municipalities. There are 9,486 cattle farms (267,551 heads), 20,295 sheep and goat farms $(3,588,489$ heads) and 16,230 pig farms (202,050 heads). These farms are registered in the BDN database (Reg CE 1760/2000 - BDN data) and their locations can be identified by geographic coordinates.

\section{Data collection}

The legislative act of the Department of Hygiene, Health and Welfare (no. 23549 of 10/11/2008, amended by no. 852 of 19/01/2009) mandates the registration of the identification number of slaughtered cattle found positive for a disease during the postmortem inspection by a veterinary officer. Lists of all positive slaughtered animals are sent to CeNRE and to the Epidemiological Veterinary Regional Centre (OEVR) on a monthly basis. An ID is given to all animals, which allows tracing information of the sex and age of animals, farm of origin and any change of property. Slaughtered cattle were aggregated into three groups according to the national classification: cows (female bovines older than 24 months), calves (6-12 months of age), baby beefs (12-24 months of age) and bulls (male bovines older than 24 months). Since only the data for the total number of slaughtered cattle were available for the Sardinian territory, the Italian data on the distribution of slaughtered cattle per age class (ISTAT) were used in this study. According to this, the percentage distribution of slaughtered cattle between 2002 and 2009 was $86.4 \%$ of calves and yearlings, $11.6 \%$ of cows and $2.0 \%$ of bulls. These percentages were constant over the time considered in this study.

\section{GIS data-layers}

Livestock farms and abattoirs, present in every municipality of Sardinia, were localized by means of GIS Mapinfo professional version 7.8 (http://www.sgsi.com/Prod_Soft_MIPro_78.asp) using geographic coordinates. Geo-referenced data of the cattle farms present in the territory of Sardinia, each coded by a specific ID, were extracted from the BDN database.

\section{Statistical analysis}

Scan statistics was used with the Bernoulli model to detect and evaluate clusters of farms defined as infected (cases) and also of "non-cases" by a variable that can be 1 or 0 , respectively. These variables may represent farms with or without disease and together they constitute the population as a whole. The Bernoulli model used by SaTScan ${ }^{\mathrm{TM}}$ is the preferred model to use when there are only two categories (e.g. positive or negative farms).

We chose a retrospective case-control study for data analysis of the distribution of infected farms in 2009. In that year, 32,685 bovines were slaughtered in Sardinia. Of these, 1,360 were found to be positive for CE with a registered average prevalence of $4.2 \%$. Of these animals, $896(66 \%)$ could be linked to one specific farm with certainty since they had lived on that farm from birth to slaughter, while $413(30 \%)$ had been transferred from one farm to another (one transfer) and $51(4 \%)$ had been shifted between three farms (two transfers). As it was impossible to know in which farm the infection was acquired, all farms having kept infected cattle were considered as suspected sources of $\mathrm{CE}$ infection. Based on this classification, 534 farms were listed as definitely infected with a further 495 suspected to possibly be so, 1,029 in all. As negative control farms, we used all Sardinian cattle farms without positive CE reports during 2009. All farms (positive and negative), the distribution of which is shown in Fig. 1, were investigated for clustering based on the permanence of infected cattle. 


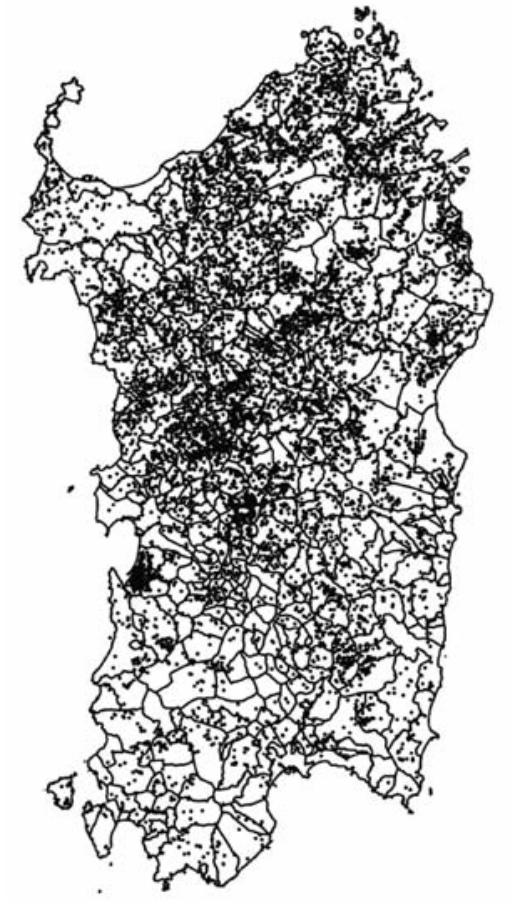

Fig. 1. Location of all 9,693 cattle farms (black) in Sardinia.

\section{Spatial analysis}

We used SaTScan ${ }^{\mathrm{TM}}$, version 8.2.1 (http://www. satscan.org/), for cluster analysis in cattle farms in the study area to highlight the core of high risk regions. This approach assumes that disease events are randomly distributed in a region as compared to outside the region under the null hypothesis, the alternative claiming elevated risk. Originally developed for point data, the method scans the studied region with a large number of circles, and detects the most likely, significant cluster(s) represented by these circle(s) (Kulldorff, 1997). The spatial analysis for clustering was represented in a Mapinfo professional, version 7.8 GIS software (Pitney Bows Mapinfo Corporation, Troy, NY, USA). The process was based on the design of a circular zone of variable size radius from zero up to a maximum specified by the user (maximum spatial cluster size) with the circle centre located on each centroid, this was fixed on points of geographic coordinates of cattle farms. The default maximum radius, which contains $50 \%$ of the population at risk inside the circle, was used. For each circular zone, the number of CE cases was compared to the expected number of cases in the population at risk. On the basis of these numbers, the likelihood function was calculated for each zone to identify potential clusters in overlapping circles. The best candidate cluster areas were evaluated. The circle with the maximum likelihood, and where

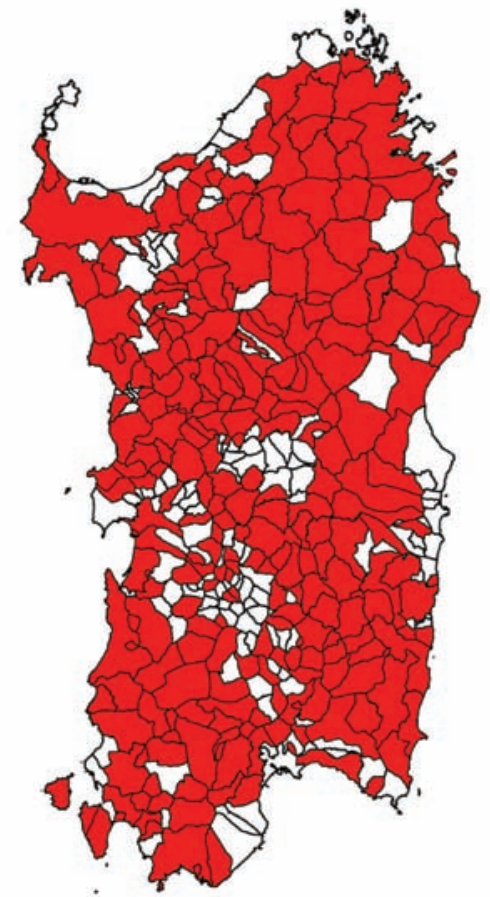

Fig. 2. Municipalities with CE-positive farms (red) in Sardinia and municipalities without (white).

there were a higher number of registered cases than expected, was designated the most likely cluster (MLC). The test was accepted to be significant when the simulated $\mathrm{P}$ value, obtained through the Monte Carlo hypothesis, was $\leq 0.05$ for the MLC allowing the null hypothesis to be rejected (Dwass, 1957; Kulldorff and Nagarwalla, 1995; Kulldorff, 1997; Boscoe et al., 2003). By performing this analysis, one primary and several secondary clusters with high likelihood values were obtained. For each cluster, a set of parameters were recovered: study period, total population, geographic coordinates, the cluster radius in $\mathrm{km}$, total cluster population (the sum of cattle farms included in the same cluster), the number of infected farms observed in the cluster, the number of expected cases in the same territory, the relative risk, the log likelihood ratio, and the $\mathrm{P}$ value.

With regard to reliability visualization we used the identification number of the farms to aggregate the cases into municipality areas and to highlight the municipality with CE cases (Fig. 2). The default maximum-size setting of $50 \%$ seldom produces usable, informative results because the reported MLC often occupies a large proportion of the study area. To address the issue of sensitivity and consistency of the results, we ran multiple scans with various maximum spatial cluster sizes (max-sizes). Specifically, we ran 50 scans using the max-size from $50 \%$ to $1 \%$ of population at risk, with a step of $1 \%$. This method allowed 
us to achieve more valid, consistent results, and to highlight the core clusters. Reliability is defined as the capacity of a test to give the same result (positive or negative, whether correct or incorrect) on repeated applications. The reliability is the consistency that a place is reported as being a high-risk area by a set of scans. It was calculated by the equation:

$$
R_{i}=C_{i} / S_{i}
$$

where $R_{i}$ is the reliability value for place $i, S$ the total number of scans and $C_{i}$ the count that place $i$ is reported in a high-risk region by these scans. The reliability value has a range from 0 to 1 , where 1 means that all scans report a place as being of high risk, while 0 means that none does. Reliability is distinct from validity, which refers to the probability that a cluster represents a true high-risk region and is measured by the cluster's statistic significance. Reliability visualization can help to highlight the core high-risk regions (Chen et al., 2008), so we calculated the reliability of the municipalities with CE-positive farms.

\section{Results}

\section{Prevalence of CE in slaughtered cattle}

A total of 1,360 out of 32,685 cattle slaughtered in Sardinia (BDN data) in 2009 were found to be positive for CE with a registered prevalence of $4.2 \%$. The highest prevalence was registered among cows $(26.2 \%)$, followed by bulls $(16.2 \%)$. In calves and yearlings, the prevalence of $\mathrm{CE}$ was considerably lower $(0.8 \%)$ (Table 1$)$.

\section{Distribution of CE-positive farms}

When accounted for at the municipality level, it was found that 246 out of 377 municipalities had CE-positive farms emphasizing that the disease is widely spread in Sardinia (Fig. 2). A total of 896 out of 1,360 cattle, found to be CE positive (66\%), had lived from birth until slaughter on the same farm. Therefore, positivity of these animals was most likely linked to the farm, the number of which amounted to 534. Of the remaining cattle, $413(30 \%)$ had been moved from the farm of birth to another, whereas 51 cattle $(4 \%)$ had been moved twice. Since movements from one location to another made it impossible to determine the farm where the animals had become infected, we considered all farms involved in the transfers $(\mathrm{n}=495)$ as suspected sources of infection. When all is taken into
Table 1. Percentage of CE positivity among cattle slaughtered in 2009.

\begin{tabular}{lccr}
\hline Cattle age-group & No. slaughtered & No. positives & $\%$ \\
\hline Cows & 3,922 & 1,027 & 26.2 \\
Bulls & 653 & 106 & 16.2 \\
Calves, baby beefs & 28,110 & 227 & 0.8 \\
Total & 32,685 & 1,360 & 4.2 \\
\hline
\end{tabular}

account, the total number of positive cattle farms in Sardinia amounts to 1,029 , i.e. 896 animals coming directly from 534 farms, which could then be termed infected, plus 495 farms, which were added due to the transfers of infected animals. Fig. 3 shows the location of the farms where the source of infection could be identified along with those where it could only be suspected.

\section{Cluster analysis}

Applying the Bernoulli case-control model accounting for the 1,029 infected or suspected farms depicted in Fig. 3 as cases and the number of non-infected farms $(8,457)$ as controls, the MLC was detected at latitude $39.47861 \mathrm{~N}$ and longitude $8.58216 \mathrm{E}$ in a centroid of $97.92 \mathrm{~km}$ radius. Within this circle, 559 cases in the population of 3,825 farms were observed. The expect-

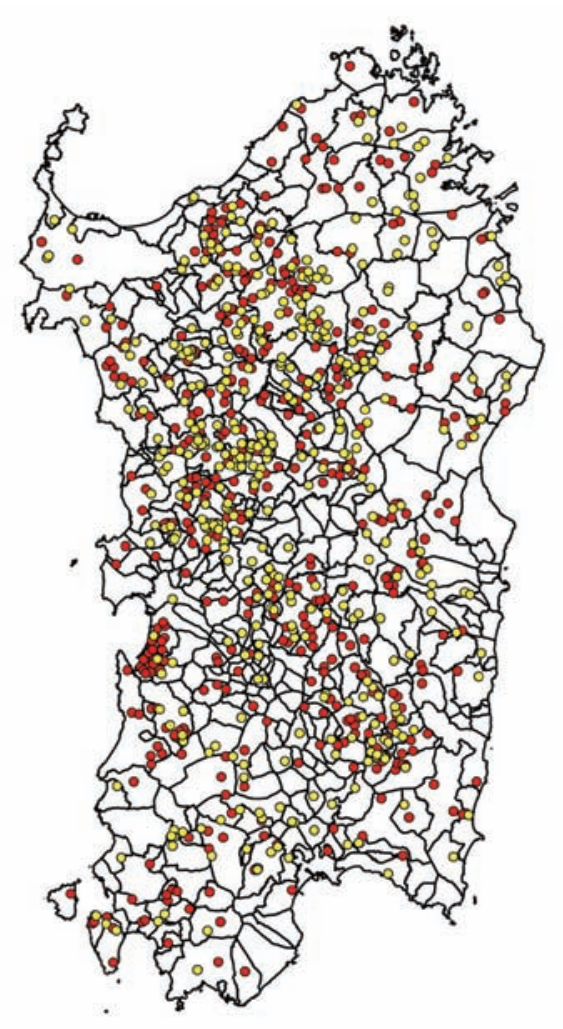

Fig. 3. Distribution and location of positive farms (red) and of suspected farms to be positive (yellow). 


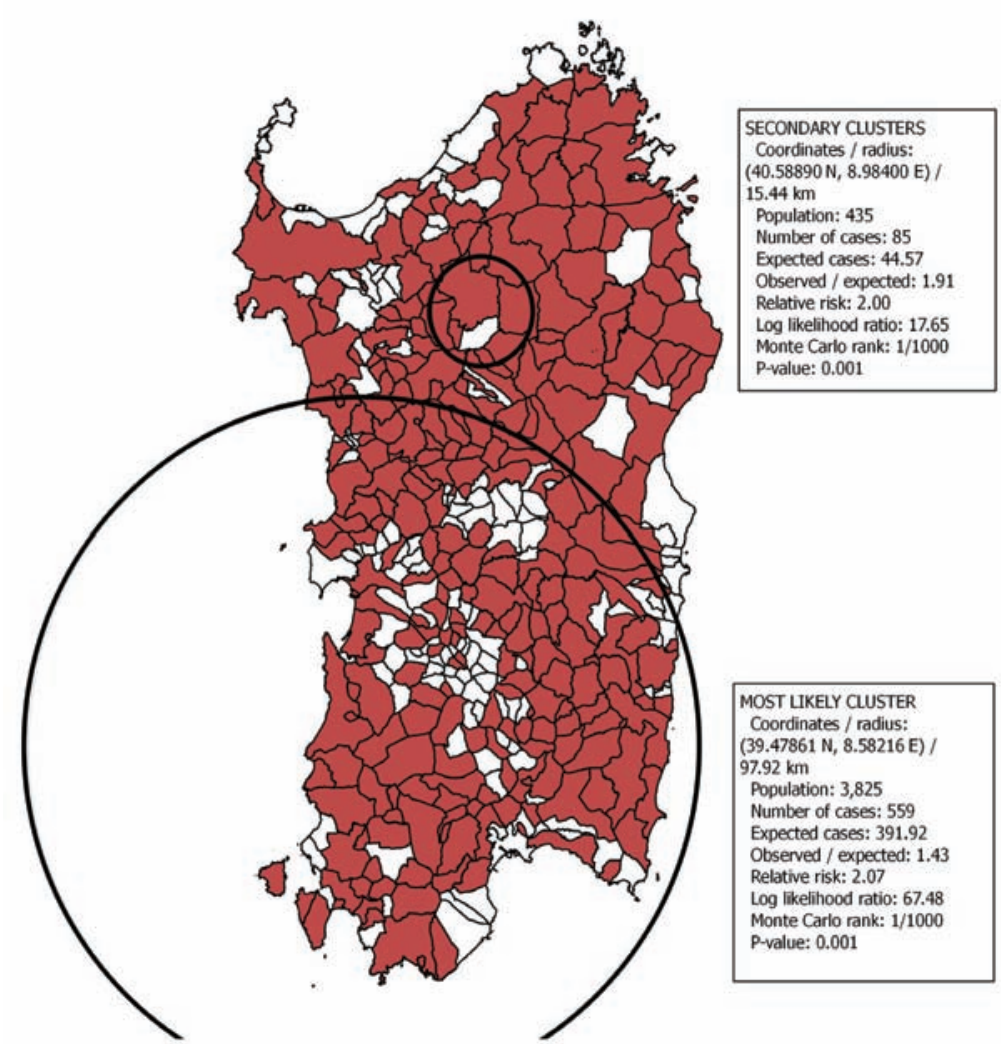

Fig. 4. Most like cluster (MLC) and a secondary cluster for the default maximum radius, i.e. containing $50 \%$ of the population at risk inside the circle.

ed number was 391.92 giving the ratio of 1.43 for observed cases to expected cases. The relative risk was 2.07 and the log likelihood ratio $67.48(\mathrm{P}=0.001)$. The analysis showed also a secondary cluster at latitude $40.58890 \mathrm{~N}$ and longitude $8.98400 \mathrm{E}$ in a centroid of $15.44 \mathrm{~km}$ radius. Within this circle, 85 cases in the population of 435 farms were observed. The expected number was 44.57 giving the ratio of 1.91 for observed cases to expected cases. The relative risk was 2.00 and the log likelihood ratio $17.65(\mathrm{P}=0.001)$ (Fig. 4).

\section{Reliability}

Since some scans of consecutive max-sizes (e.g. sizes from $50 \%$ to $42 \%$ ) produced similar results, we selected only one to represent the others. We used only significant clusters $(\mathrm{p}<0.05)$ to calculate the reliability.
The scans from $50 \%$ to $1 \%$ of population at risk reported as small $\mathrm{P}$ values as 0.001 for all MLCs and for the most part of the secondary clusters (Table 2). We also plotted the 50 results in a map matrix with each map displaying the significant, high-risk clusters found and visually verified that the selected 18 results could represent the others in terms of the location and shape of the clusters. Fig. 5 displays the selected 18 results that reasonably represented all the 50 results. Fig. 6 highlights the core high-risk regions (circles A, $\mathrm{B}, \mathrm{C}$ and D). For the municipalities depicted in darkgreen colour inside the circles, we could reject the null hypothesis for all the max-sizes, while regions with low reliability (shown in light-green colour and blue) we were unable to do so, as the max-sizes were reduced. Municipalities depicted in white contain only farms without CE-positive cases.

Table 2. P values of the most likely and secondary clusters.

\begin{tabular}{lllllll}
\hline Scans & $17-50 \%$ & $7-16 \%$ & $5-6 \%$ & $4 \%$ & $2-3 \%$ & $1 \%$ \\
\hline MLC P value & 0.001 & 0.001 & 0.001 & 0.001 & 0.001 & 0.001 \\
Sc P value & & & & & $0.001(2)$ & \\
No. of clusters & $0.001(1)$ & $0.001(2)$ & $0.001(3)$ & $0.001(2)$ & $0.002(1)$ & $0.002(4)$ \\
& & & & $0.002(1)$ & $0.042(1)$ & $0.042(2)$ \\
\hline
\end{tabular}




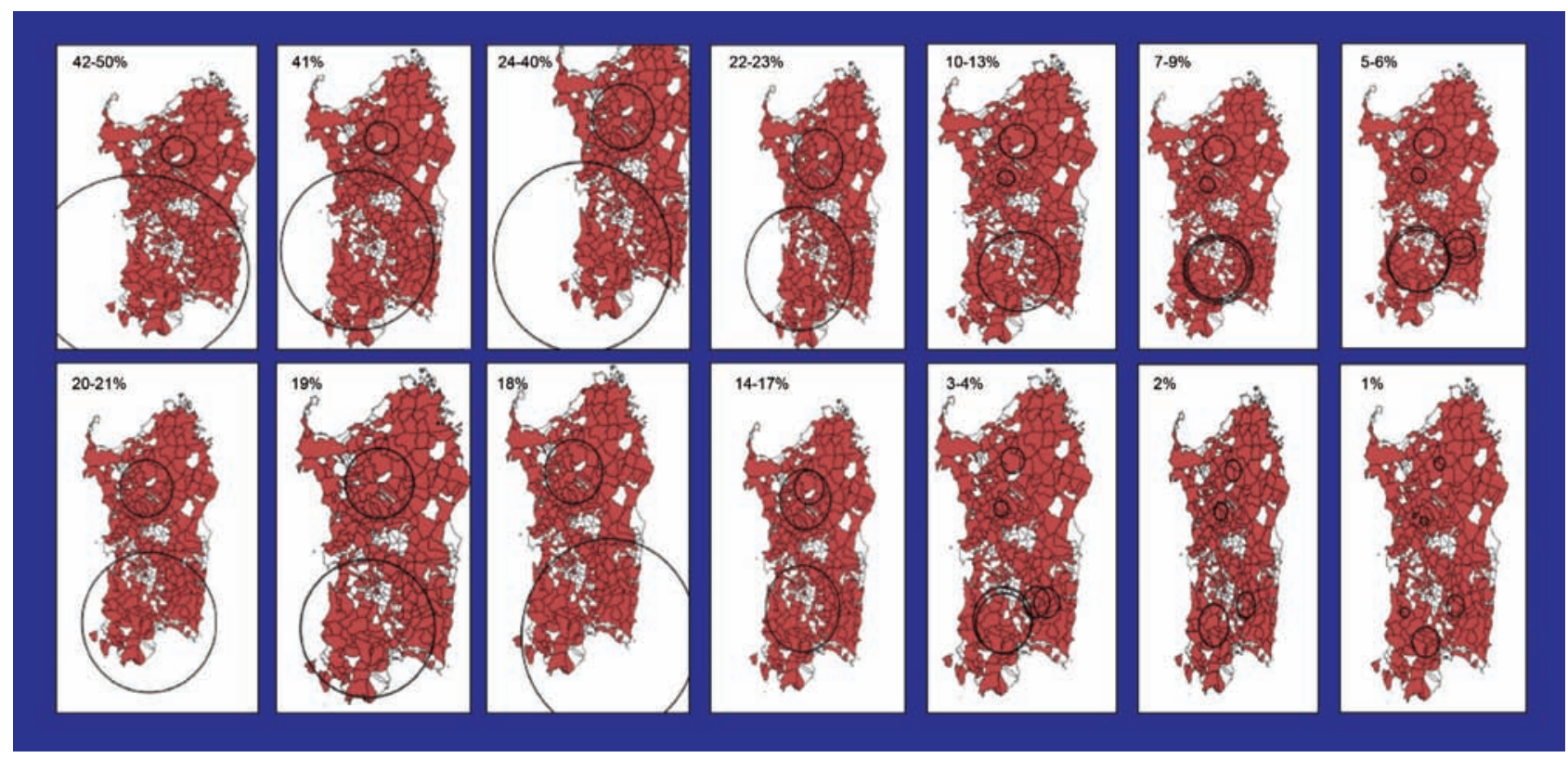

Fig. 5. The maps display 18 SaTScan results. Only significant clusters $(\mathrm{P}<0.05)$ are displayed.

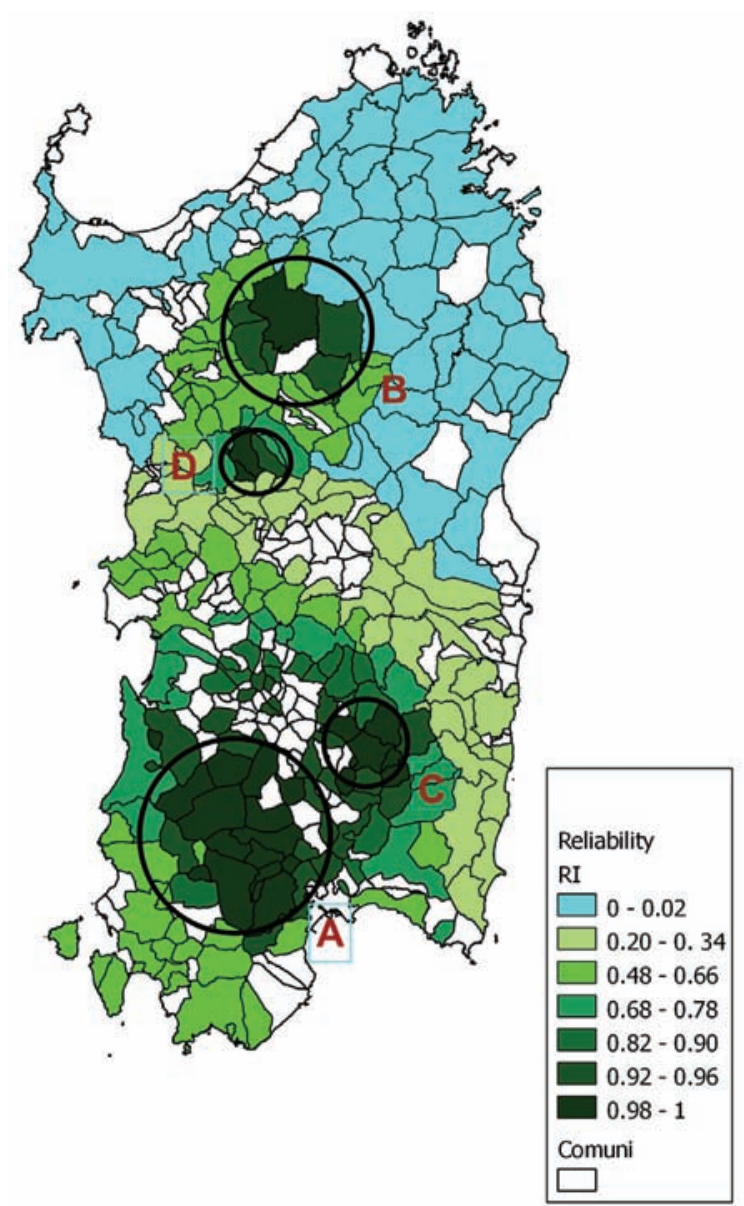

Fig. 6. The map displays the reliability values calculated from 18 scans. Four core clusters (A, B, C and D) are highlighted. The dark green regions are consistently reported as being of highrisk, while the white areas represent municipalities free from cystic echinococcosis.

\section{Discussion}

An unprecedented detailed picture on the distribution of CE in Sardinian cattle was obtained showing a high prevalence of $\mathrm{CE}$ in all cattle older than 24 months (cows and bulls) slaughtered. Moreover, the integration of data with the GIS software made it possible to create maps enabling the identification of clusters of the disease including risk analysis. This analysis worked well in cattle when the animals were investigated one by one. However, bovines are not as important as sheep for the E. granulosus lifecycle, and particularly not in Sardinia, as they are usually only slaughtered in one of only a few large, modern and efficient abattoirs, where the offal is destroyed and where the presence of canids is strictly forbidden. Therefore, it is highly unlikely that dogs or other canids had the opportunity to feed on cattle carcasses in these places (Cringoli et al., 2007). Moreover, only a very low prevalence of fertile bovine cysts $(2.6 \%)$ has been reported in Sardinia (Scala et al., 2004).

A possible cause of the clustering results could be the high population density (PD) of sheep in the the high-risk regions, i.e. the municipalities with high reliability ( $>0.98$ ) shown in Fig. 6 (circles A, B, C and D). These municipalities present a higher number and a higher PD of sheep and cattle than the mean of the municipalities in Sardinia as a whole (BDN data). These municipalities are characterised by free-range bovine breeding, often associated with sheep breeding, where cattle and sheep usually graze in the same pastures. Importantly, the average number of cattle per 
municipality here ( 923 with a PD of 12 heads $/ \mathrm{km}^{2}$ ) is higher than the Sardinian average (609 with 8 heads $/ \mathrm{km}^{2}$ ). The data with respect to sheep indicate a high mean of 19,700 heads per municipality with a PD of 233 heads $/ \mathrm{km}^{2}$, while the Sardinian mean is 8,800 with a PD of 164 heads $/ \mathrm{km}^{2}$.

It should be noted that sheep are the intermediate host of choice for E. granulosus and that sheep are often slaughtered at home and their offal then often used to feed dogs. Furthermore, carcasses of sheep which die on the pastures are not disposed of but generally left where they have fallen (Scala et al., 2004). These facts are in correspondence with epidemiological studies from all over the world which show that sheep is the key reservoir for E. granulosus (e.g. Romig, 2003; Scala et al., 2004; Battelli et al., 2007; Daryani et al., 2007). However, although cattle might not have a significant role in the persistence of this important zoonosis, it might still be useful as an indicator of CE infection (Rinaldi et al., 2008). An accurate, epidemiological GIS analysis would show the precise location of sheep farms hosting infected animals. It would also enable a study of the correlation of animal and human cases, which is a necessary condition to target preventive measures and to plan a proper CE control programme.

\section{Conclusions}

The data-flow model used for cattle provides useful epidemiological information with respect to CE. If applied to sheep, which have a higher relevance in epidemiology of this zoonosis, it would provide even better and more relevant information. A reliable assessment of the degree of intervention needed in Sardinia would be possible if the precise localization of farms hosting infested animals could be listed and analysed in combination with the incidence data.

\section{References}

Battelli G, Guazzetti S, Micagni G, Ostanello F, 2007. Bovine echinococcosis in non-endemic area of northern Italy: an example of integrated analysis of passive surveillance data. Proceedings of the 22th International Coongress of Hydatidology, Athens (Greece), 15-19 May 2007, pp. 131-133. Boscoe FP, McLaughlin C, Schymura MJ, Kielb CL, 2003. Visualization of the spatial scan statistic using nested circles. Health Place 9, 273-277.

Budke CM, Deplazes P, Torgerson PR 2006. Global socioeconomic impact of cystic echinococcosis. Emerg Infect Dis 12, 296-303.

Chen J, Roth RE, Naito AT, Lengerich EJ, MacEachren AM,
2008. Int J Health Geogr 7, 57.

Craig PS, McManus DP, Lightowlers MW, Chabalgoity JA, Garcia HH, Gavidia CM, Gilman RH, Gonzalez AE, Lorca M, Naquira C, Nieto A, Schantz PM, 2007. Prevention and control of cystic echinococcosis. Lancet Infect Dis 7, 385-394.

Cringoli G, Rinaldi L, Musella V, Veneziano V, Maurelli MP, Di Pietro F, Frisiello M, Di Pietro S, 2007. Geo-referencing livestock farms as tool for studying cystic echinococcosis epidemiology in cattle and water buffaloes from southern Italy. Geospat Health 2, 105-111.

Daryani A, Alei R, Arab R, Sharif M, Dehghan MH, Ziaei H 2007. The prevalence, intensity and viability of hydatid cysts in slaughtered animals in the Ardabil province of Northwest Iran. J Helminthol 81, 13-17.

Dwass M, 1957. Modified randomization tests for nonparametric hypotheses. Ann Math Stat 28, 181-187.

Garippa G, 2006. Updates on cystic echinococcosis (CE) in Italy. Parassitologia 48, 57-59.

Jenkins DJ, Romig T, Thompson RC, 2005. Emergence/re-emergence of Echinococcus spp.: a global update. Int J Parasitol 35, 1205-1219.

Kulldorff M, 1997. A spatial scan statistic. Commun Stat ATheor 26, 1481-1496.

Kulldorff M, Nagarwalla N, 1995. Spatial disease clusters: detection and inference. Stat Med 14, 799-810.

McManus DP, Zhang W, Li J, Bartley PB, 2003. Echinococcosis. Lancet 18, 1295-1304.

Moro P, Schantz PM, 2006. Cystic echinococcosis in the Americas. Parasitol Int 55, 181-186.

Rinaldi L, Maurelli MP, Veneziano V, Capuano F, Perugini AG, Cringoli G, 2008. The role of cattle in the epidemiology of Echinococcus granulosus in an endemic area of southern Italy. Parasitol Res 103, 175-179.

Romig T, 2003. Epidemiology of echinococcosis. Langenbecks Arch Surg 388, 209-217.

Romig T, Dinkel A, Mackenstedt U, 2006. The present situation of echinococcosis in Europe. Parasitol Int 55, 187-191.

Scala A, Canu S, Tanda B, Basciu M, Polinas L, Sanna Coccone GN, Pilloni S, Canu S, Varcasia A, Garippa A, 2004. An epidemiological and biomolecular survey of cystic echinococcosis in cattle in Sardinia. Parassitologia 46, 443-444.

Scala A, Garippa G, Varcasia A, Tranquillo VM, Genchi C, 2006. Cystic echinococcosis in slaughtered sheep in Sardinia (Italy). Vet Parasitol 135, 33-38.

Schantz PM, Chai J, Eckert J, Jenkins DJ, Macpherson CNL, Thakur A. 1995. Epidemiology and control of hydatid disease. In: Thompson RCA, Lymbery AJ (eds) Echinococcus and hydatid, disease. CAB International, Wallingford, UK, pp. 233-331.

Seimenis A, 2003. Overview of the epidemiological situation on echinococcosis in the Mediterranean region. Acta Trop 85, 191-195. 